$p_{2}, \cdots, p_{7}$, and of orders 48,128 , and 336 at $A, B$, and $C$, respectively; and so on.

\title{
REFERENCES
}

A. B. Coble

1. The ten nodes of the rational sextic and the Cayley symmetroid, American Journal of Mathematics, vol. 41 (1919), pp. 243-265.

2. Algebraic Geometry and Theta Functions, American Mathematical Society Colloquium Publications, vol. 10, New York, 1929.

Giuse pre Pompili

1. Sulle trasformazioni cremoniane del piano che posseggono una curva di punti uniti, Rendiconti del Seminario Matematico della Università di Roma, (4), vol. 2 (1938), pp. 47-87.

UNIVERSITY OF ILLINOIS

\section{A NOTE ON THE REDUCTION OF GENTZEN'S CALCULUS LJ*}

H. B. CURRY

The reduction given by Gentzen $\dagger$ of his calculus $\mathrm{LJ}$ to the modified Heyting calculus LHJ is somewhat involved because he reduces everything to the axioms without assuming any knowledge of the calculus. By the use, however, of certain general theorems it is possible to simplify the reduction. The purpose of this note is to present an alternative reduction based on this principle. Although this new reduction may, if all the assumptions used are proved in detail from the axioms, conceivably be longer than Gentzen's, yet the formulas and principles established at the beginning (in $\$ \$ 1-4$ below) are for the most part well known (or at least of some interest on their own account), and in terms of these the reduction (in §5) is almost immediate.

The new method has the further merit of showing, if we take the axioms of LHJ as a basis, that the schemes for implication follow from the axioms for implication only $\ddagger$ and that those for conjunction, negation, and the quantifiers, respectively, involve only the axioms for implication and those for the operation concerned. $\$$ It

* Presented at a joint meeting of the Society and the Association for Symbolic Logic, December 29, 1937.

$\dagger$ G. Gentzen, Untersuchungen über das logische Schliessen, Mathematische Zeitschrift, vol. 39 (1934), pp. 417-428.

¥ The scheme "Verdünnung im Sukzedens" is essentially a scheme for negation.

$\S$ This statement requires that the formula 1.42 (below) be postulated as axiom of $\mathrm{LHJ}$; to derive 1.42 from $\mathrm{LHJ}$ as it stands requires properties of conjunction. 
then follows from Gentzen's principal theorem that if a formula can be established in the Heyting calculus at all, it can be established on the basis of those axioms of the calculus $\mathrm{LHJ}$ which concern implication and the other operations, if any, which actually appear in the formula. $\uparrow$

The notation of this paper is the same as Gentzen's except as follows: For conjunction $\wedge$ will be used instead of $\&$; the usual notation $(x)$ will be used for the universal quantifier; and ordinary (italic) letters, instead of German ones, will be used for unspecified expressions of the calculus. On the use of dots see my paper On the use of dots as brackets in logical expressions. \& Certain formulas and theorems which are not necessary for the immediate purpose are preceded by *; some of these are useful for certain generalizations mentioned at the end.

1. Preliminary formulas and principles. The first step is to derive the following formulas and principles:

1.1. Properties of implication.

1.10. $\frac{A, A \supset B}{B}$.

*1.11. $A \supset A$.

1.12. $A \supset$. $B \supset A$.

1.13. $A \supset . A \supset B: \supset: A \supset B$.

1.14. $A \supset . B \supset C: \supset: B \supset . A \supset C$.

1.15. $B \supset C . \supset: A \supset B . \supset . A \supset C$.

1.16. $A \supset B . \supset: B \supset C . \supset . A \supset C$.

1.17. $\frac{A \supset B, B \supset C}{A \supset C}$.

1.2. Properties of conjunction and alternation. These are given, partly in dual form, as follows:

1.21. $A \wedge B . \supset . A, A \wedge B . \supset . B ; B . \supset . A \vee B, A . \supset . A \vee B$.

1.22. $\frac{A, B}{A \wedge B}$.

1.23. $C \supset A . \wedge . C \supset B: \supset: C . \supset . A \wedge B$, and $A \supset C . \wedge . B \supset C: \supset: A \vee B . \supset . C$.

$\dagger$ This result appears to be generally known; but I am acquainted with no published proof of it. For partial results of the same nature see D. Hilbert and P. Bernays, Grundlagen der Mathematik, vol 1, 1934, p. 71; also I. Johansson, Compositio Mathematica, vol. 4 (1937), p.131. Added in proof: See also M. Wajsberg, Untersuchungen über den Aussagenkalkul von A. Heyting, Wiadomošci Matematyczne, vol. 46 (1938), pp. 45-101.

¥ Journal of Symbolic Logic, vol. 2 (1937), pp. 26-28. 


$$
\text { *1.24. } \begin{aligned}
\frac{A}{A \wedge C . \supset . B \wedge C}, & \frac{B \supset A}{B \vee C . \supset . A \vee C}, \\
\frac{A \supset B}{C \wedge A . \supset . C \wedge B} ; & \frac{B \supset A}{C \vee B . \supset . C \vee A} .
\end{aligned}
$$

*1.25. $A \supset$ ว $B \supset . A \wedge B$.

*1.26. $A . \wedge . A \supset B . \supset . B$.

*1.3. Formal algebraic properties of $\wedge$ and $\boldsymbol{\vee}$, that is, the commutative, associative, and distributive laws and the laws of tautology.

1.4. Properties of quantifiers.

1.40. If $F a$ holds for a free variable $a$ not in $F$, then $(x) F x$ holds. 1.41. $(x) F x . \supset . F a$, and $F a . \supset .(\exists x) F x$.

1.42. If $x$ does not occur in $A$,
(x) . $A \supset F x: \supset: A . \supset .(x) F x$,
$(x) . F x \supset A: \supset:(\exists x) F x . \supset . A$.

1.5. Properties of negation.

1.51. $A \supset$. ᄀ $A$ ว $B . \dagger$

1.52. $\frac{T}{A \supset \neg T . \supset .\urcorner A} \cdot$

*1.54. $A \supset B$. . . . ᄀ $B$ ว $A$.

2. A general substitution theorem. Using these we may, following S. MacLane, $\ddagger$ prove a general substitution theorem as follows:

2.1. First, let us say that an operation $\phi$ on $A$ is positive when

$$
\frac{A \supset B}{\phi A \supset \phi B}
$$

is a valid scheme, negative when

$$
\frac{A \supset B}{\phi B \supset \phi A}
$$

$\dagger 1.51$ is a weaker form of $A \supset . \neg A \supset B$, which is essentially equivalent to 1.53 (and, by 1.14 , is equivalent to the axiom $\neg A . \supset . A \supset B$ ). However, 1.51 is true in the Minimalkalkul of Johansson (Compositio Mathematica, vol.4 (1936), pp.119-136), while the stronger principles are not. Hence I shall use the stronger principle 1.53 only in the derivation of the scheme DS (5.43) where 1.51 does not suffice. This establishes all the schemes except DS for the calculus LM (Johansson, loc. cit., §5).

$\ddagger$ Abgekürzte Beweise im Logikkalkul, Inaugural-dissertation, Göttingen 1934, p. $28 \mathrm{ff}$. A similar theorem appears in J. Herbrand, Recherches sur la Thêorie de la Démonstration, Warsaw, 1930, §3.2. 
is a valid scheme. Then by $1.15,1.24$ the operations $C \supset, \dagger C \wedge, \wedge C$, $C \vee, \vee C$ are positive; on the other hand by 1.16, $1.54 \supset C$ and \urcorner are negative. $\ddagger$

2.2. Suppose now we have an operation $\psi$ which is compounded of certain operations $\phi_{1}, \phi_{2}, \cdots, \phi_{n}$ in the sense that

$$
\psi A=\phi_{n}\left(\phi_{n-1}\left(\cdots\left(\phi_{1} A\right) \cdots\right)\right) .
$$

Then if each of the $\phi_{i}$ is either positive or negative, $\psi$ will be positive or negative according as the number of negative $\phi$ 's is even or odd. (This is readily proved by induction.)

2.3. Hence suppose that we have a provable formula which is constructed from a particular instance of a formula $A$ by the operations listed in 2.1 ; then if the number of negative operations is even, that particular instance of $A$ may be replaced by any $B$ such that $A \supset B$ holds; if the number of negative operations is odd, it may be replaced by any $C$ such that $C \supset A$ holds.

3 . Further properties of $\phi$. Let us now consider two further properties of an operation $\phi$ as follows:

I. $\phi A \wedge \phi B . \supset . \phi(A \wedge B)$.

II. $(x) \phi(F x)$. ว . $\phi((x) F x)$.

Concerning these we have the following theorems:

3.1. If the $\phi_{i}$ of 2.2 are all positive and have property I, then $\phi$ also has property $\mathrm{I}$.

Proof. Let $\psi_{k}$ be defined by

$$
\psi_{1} A=\phi_{1} A, \quad \psi_{k+1} A=\phi_{k+1}\left(\psi_{k} A\right) .
$$

Then $\psi_{1}$ has property I. Suppose that $\psi_{k}$ does; then by 1.1 , and since $\phi_{k+1}$ has property I,

$$
\begin{aligned}
\psi_{k+1} A \wedge \psi_{k+1} B & . \supset \phi_{k+1}\left(\psi_{k} A\right) \wedge \phi_{k+1}\left(\psi_{k} B\right) \\
& . \supset . \phi_{k+1}\left(\psi_{k} A \wedge \psi_{k} B\right) \\
& . \supset . \phi_{k+1}\left(\psi_{k}(A \wedge B)\right)
\end{aligned}
$$

since $\psi_{k}$ has property I and $\phi_{k+1}$ is positive. The transitive property of $\supset$ holds by 1.17 . Hence $\psi_{k+1}$ has property I, and the theorem follows by induction.

$\dagger$ By this I mean the operation converting $A$ into $C \supset A$, and similarly for the other cases. The $C$ 's may be different in the different instances of the operators.

$\ddagger$ The fact that $\neg$ is a negative operation is not used in establishing the schemes in $\$ 5$ below. Hence 1.54 is marked with *; similarly for 1.24 . 
3.2. If the $\phi_{i}$ of 2.2 are all positive and have property II, then $\psi$ also has property II. (The proof is similar to that of 3.1.)

3.3. In the following we shall need only the special case of the foregoing theorems where $\phi_{i}$ is $C_{i} \supset$, with the additional proviso, in the case of II, that $C_{i}$ does not contain $x$. In this case the hypotheses regarding the $\phi_{i}$ are satisfied by 1.23 (in case I) and 1.42 (in case II).

4. Chain implications. Concerning formulas of the form

$$
A_{1} \supset A_{2} \supset \ldots \supset . A_{n} \supset B
$$

we have the following theorems:

4.1. From the axioms for implication (1.1) it follows that the $A_{i}$ may be permuted in any manner, that repetitions may be cut out, and that new $A$ 's may be inserted at pleasure.

*4.2. From the axioms for implication and conjunction, it follows that (1) is equivalent to

$$
A_{1} \wedge A_{2} \wedge \cdots \wedge A_{n} \cdot \supset . B .
$$

(The proof would require some of the starred propositions of $\$ 1$.)

5. Derivation of the schemes. Suppose now that we replace the sequence

$$
A_{1}, A_{2}, \cdots, A_{n} \rightarrow B
$$

of Gentzen by the corresponding formula (1) of 4.1 ; with the proviso that where Gentzen has a sequence with a void consequent, a consequent $\Lambda$, where $\Lambda$ is $\neg T$ and $T$ is some provable formula, must first be supplied. Then the limitations of the calculus LJ insure that every sequence of an "LJ Herleitung" is of the kind just considered. We shall now see that Gentzen's schemes go over into valid principles of inference in the calculus LHJ. In this deduction I shall use D for "Verdünnung," Z for "Zusammenziehung," and $T$ for "Vertauschung." Then the schemes are established as follows:

5.1. Schemes for $\supset$.

5.11. DA, ZA, TA, FES by 4.1 .

5.12. Schnitt by 2.3 .

5.13. FEA thus: by induction and $1.15,1.17$,

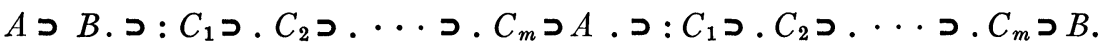
Hence if $C_{1} \supset C_{2} \supset \ldots$. $\supset C_{m} \supset A$ holds, we have, by 1.14 and 1.10,

$$
A \supset B . \supset . C_{1} \supset . C_{2} \supset \ldots \text { ว . . } C_{m} \supset B .
$$

The scheme is now reduced to Schnitt. 
5.2. Schemes for $\wedge$ and $\boldsymbol{\vee}$.

5.21. UES by $1.23,3.1,3.3$.

5.22. OEA by 1.23 immediately.

5.23. UEA and OES by 1.21 and 2.3 .

5.3. Schemes for quantifiers.

5.31. AES by 1.40, 3.2, 3.3.

5.32. EEA by $1.40,1.42,1.10$.

5.33. AEA and EES (dually) by 1.41 and 2.3.

5.4. Schemes for negation.

5.41. NEA thus:

$$
\begin{aligned}
& \underline{C_{1} \supset . C_{2} \supset \ldots \text { ว } . C_{m} \supset A}(1.51,2.3) \\
& \underline{C_{1} \supset . C_{2} \supset \ldots \cdots \supset C_{m} \supset . \neg A \supset \Lambda} \text { (4.1) }
\end{aligned}
$$

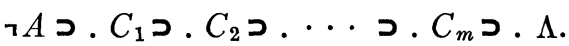

5.42. NES thus:

$$
\begin{aligned}
& \frac{A \supset . C_{1} \supset . C_{2} \supset \ldots \cdots \supset C_{m} \supset \neg T}{C_{1} \supset . C_{2} \supset \ldots \cdots \supset . C_{m} \supset . A \supset \neg T}(4.1) \\
& C_{1} \supset . C_{2} \supset \ldots \ldots . C_{m} \supset . \neg A .
\end{aligned}
$$

\subsection{DS by $1.53,2.3$.}

6. Conclusion. It may be worth remarking that by these same methods many of Gentzen's schemes may be established without the restriction to not more than one element in the consequent; the consequent must then be taken as a logical sum. In fact by the use of the formulas marked with a ${ }^{*}$ this can be done for all the schemes which do not involve quantifiers except NES and FES. $†$ These schemes are in general invalid. NES, in fact, leads to the law of excluded middle (Gentzen, loc. cit., p. 193); while FES leads (for void $\Gamma$ ) to the scheme

$$
\frac{A . \supset . C \vee B}{C . \supset . A \vee B}
$$

which is not valid.

\section{The Pennsylvania State College}

$\dagger$ In the minimal calculus the generalized NEA is also invalid. 